\title{
On uniform exponential splitting for noninvertible evolution operators in Banach Spaces
}

Claudia Luminița Mihiţ, Codruţa Simona Stoica, and Mihail Megan

\begin{abstract}
The paper considers the general concept of uniform exponential splitting as a generalization of uniform exponential dichotomy property for evolution operators in Banach spaces.

Two characterizations in terms of integral inequalities of Datkotype respectively Lyapunov functions for uniform exponential splitting of a noninvertible evolution operator with respect to invariant projections families are obtained.
\end{abstract}

AMS Subject Classification (2000). 34D05, 34D09

Keywords. Uniform exponential splitting, uniform exponential dichotomy, evolution operators

\section{Introduction}

The notion of exponential dichotomy introduced by O. Perron in 1930 ([13]) is one of the most important properties in the qualitative theory of the asymptotic behaviors of evolution operators in finite and infinite dimensional spaces (see [1]-[8] and the references therein).

While most of the research on exponential dichotomies has been devoted to invertible dynamical systems (see e.g [10], [12]), there are only a few papers dealing with the general noninvertible case (see e.g [6], [9], [14]). 
The study of noninvertible systems is of great importance and in this sense we point out the paper of B. Aulbach and J. Kalkbrenner [1], where is introduced the notion of exponential forward splitting, motivated by the fact that there are differential equations whose backward solutions are not guaranteed to exist. This approach is of interest in applications, see for example, random dynamical systems, generated by random parabolic equations, are not invertible ( for more details see L. Zhou et al. [15]).

The most important dichotomy concept used in the qualitative theory of dynamical systems is the uniform exponential dichotomy. In some situations, particularly in the nonautonomous setting the concept of uniform exponential dichotomy is too restrictive and it is important to consider more general behaviors (see for instance [3], [8], [11]).

Two different perspectives can be identified to generalize the concept of uniform exponential dichotomy, one can define dichotomies that depends on the initial time ( and therefore are nonuniform ) and, on the other hand, one can consider growth rates which not imply an exponential dichotomy behavior, in particular the exponential splitting.

Based on this fact, the purpose of this paper is to give two characterizations in terms of integral inequalities of Datko-type respectively Lyapunov functions for uniform exponential splitting of an evolution operator with respect to invariant projections families. As particular cases, characterizations for uniform exponential dichotomy are obtained.

We note that we consider evolution operators which are not supposed to be invertible ( in particular, evolution operators which are not generated by differential equations ) and moreover, the time variable is considered on the half-line.

\section{Uniform exponential splitting}

Let $X$ be a real or complex Banach space and $\mathcal{B}(X)$ the Banach algebra of all bounded linear operators on $X$. The norms on $X$ and on $\mathcal{B}(X)$ will be denoted by $\|\cdot\|$.

Also, we denote

$$
\Delta=\left\{(t, s) \in \mathbb{R}_{+}^{2}: t \geq s\right\} .
$$

We recall that an operator valued function $\Phi: \Delta \rightarrow \mathcal{B}(X)$ is called an evolution operator on $X$ if $\Phi(t, t)=I$ (the identity operator on $X$ ) and $\Phi\left(t, t_{0}\right)=\Phi(t, s) \Phi\left(s, t_{0}\right)$, for all $t \geq s \geq t_{0} \geq 0$.

If $P: \mathbb{R}_{+} \rightarrow \mathcal{B}(X)$ is a family of projections on $X$ (i.e. $P(t)^{2}=P(t)$, for every $t \geq 0$ ) which is invariant for the evolution operator $\Phi: \Delta \rightarrow \mathcal{B}(X)$ 
(i.e. $P(t) \Phi(t, s)=\Phi(t, s) P(s)$, for all $(t, s) \in \Delta)$, then $Q: \mathbb{R}_{+} \rightarrow \mathcal{B}(X)$ defined by $Q(t)=I-P(t)$ is a family of projections on $X$ (which is called the complementary family of projections of $P$ ) and it is also invariant for $\Phi$.

In what follows, we consider an evolution operator $\Phi: \Delta \rightarrow \mathcal{B}(X)$ and a family of projections $P: \mathbb{R}_{+} \rightarrow \mathcal{B}(X)$ which is invariant for $\Phi$.

Also, for $(t, s) \in \Delta$, we will denote

$$
\Phi_{P}(t, s)=\Phi(t, s) P(s) \quad \text { and } \quad \Phi_{Q}(t, s)=\Phi(t, s) Q(s) .
$$

Definition 2.1. We say that the pair $(\Phi, P)$ admits an uniform exponential splitting if there exist the real constants $N \geq 1$ and $\alpha<\beta$ such that:

$$
\left(u e s_{1}\right) \quad\left\|\Phi_{P}(t, s) x\right\| \leq N e^{\alpha(t-s)}\|P(s) x\| ;
$$

and

$$
\left(u e s_{2}\right) \quad e^{\beta(t-s)}\|Q(s) x\| \leq N\left\|\Phi_{Q}(t, s) x\right\|,
$$

for all $(t, s, x) \in \Delta \times X$.

The constants $\alpha$ and $\beta$ are called splitting rates.

In particular, if $\alpha<0<\beta$, then we say that $(\Phi, P)$ has an uniform exponential dichotomy.

Remark 2.1. If the pair $(\Phi, P)$ has an uniform exponential dichotomy, then it also admits an uniform exponential splitting. The following example shows that the converse implication is not valid.

Example 2.1. We consider $X=l^{\infty}(\mathbb{N}, \mathbb{R})$ the Banach space of bounded real-valued sequences, endowed with the norm

$$
\|x\|=\sup _{n \in \mathbb{N}}\left|x_{n}\right|, \quad x=\left(x_{0}, x_{1}, \ldots x_{n}, \ldots\right) \in X .
$$

Let $P: \mathbb{R}_{+} \rightarrow \mathcal{B}(X)$ be the family of projections, defined by

$$
P(t)\left(x_{0}, x_{1}, \ldots\right)=\left(0, x_{1}, 0,0, x_{4}, 0,0, \ldots\right) .
$$

The complementary family of $P$ is $Q: \mathbb{R}_{+} \rightarrow \mathcal{B}(X)$,

$$
Q(t)\left(x_{0}, x_{1}, \ldots\right)=\left(x_{0}, 0, x_{2}, x_{3}, 0, x_{5}, x_{6}, \ldots\right) .
$$

We define $\Phi: \Delta \rightarrow \mathcal{B}(X)$ by

$$
\Phi(t, s) x=\left(x_{0} e^{4\left(t^{2}-s^{2}\right)}, x_{1} e^{2(t-s)}, x_{2} e^{4(t-s)}, x_{3} e^{4\left(t^{2}-s^{2}\right)}, x_{4} e^{2(t-s)}, \ldots\right),
$$

for all $(t, s) \in \Delta$ and all $x=\left(x_{0}, x_{1}, x_{2}, \ldots\right) \in X$. 
We observe that

$$
\Phi_{P}(t, s) x=\left(0, x_{1} e^{2(t-s)}, 0,0, x_{4} e^{2(t-s)}, \ldots\right)
$$

and

$$
\Phi_{Q}(t, s) x=\left(x_{0} e^{4\left(t^{2}-s^{2}\right)}, 0, x_{2} e^{4(t-s)}, x_{3} e^{4\left(t^{2}-s^{2}\right)}, 0, \ldots\right),
$$

for all $(t, s) \in \Delta$ and $x=\left(x_{0}, x_{1}, x_{2}, \ldots\right) \in X$.

From here we obtain that

$$
\left\|\Phi_{P}(t, s) x\right\| \leq e^{2(t-s)}\|P(s) x\|
$$

and

$$
e^{4(t-s)}\|Q(s) x\| \leq\left\|\Phi_{Q}(t, s) x\right\|,
$$

for all $(t, s, x) \in \Delta \times X$.

Thus, $(\Phi, P)$ admits an uniform exponential splitting with the splitting rates $\alpha=2<4=\beta$.

If we suppose that $(\Phi, P)$ has an uniform exponential dichotomy then there exist $N \geq 1, \alpha>0$ such that

$$
\left\|\Phi_{P}(t, s) x\right\| \leq N e^{-\alpha(t-s)}\|P(s) x\| \text {, for all }(t, s, x) \in \Delta \times X .
$$

In particular for $s=0$ and $x=(0,1,0,0,1,0, \ldots)$ we obtain

$$
e^{2 t} \leq N e^{-\alpha t}, \text { for all } t \geq 0
$$

which yields to a contradiction.

Remark 2.2. The pair $(\Phi, P)$ admits an uniform exponential splitting if and only if there exist the constants $N \geq 1, \alpha, \beta \in \mathbb{R}, \alpha<\beta$ with the properties:

$$
\left(u e s_{1}^{\prime}\right) \quad\left\|\Phi_{P}\left(t, t_{0}\right) x\right\| \leq N e^{\alpha(t-s)}\left\|\Phi_{P}\left(s, t_{0}\right) x\right\| ;
$$

and

$$
\left(u e s_{2}^{\prime}\right) \quad e^{\beta(t-s)}\left\|\Phi_{Q}\left(s, t_{0}\right) x\right\| \leq N\left\|\Phi_{Q}\left(t, t_{0}\right) x\right\|,
$$

for all $t \geq s \geq t_{0} \geq 0$ and all $x \in X$.

Proposition 2.1. The pair $(\Phi, P)$ admits an uniform exponential dichotomy if and only if there exists $\nu>0$ such that:

$\left(\right.$ ued $\left._{1}\right)\left\|\Phi_{P}(t, s) x\right\| \leq N e^{-\nu(t-s)}\|P(s) x\| ;$

$\left(u_{e} d_{2}\right) e^{\nu(t-s)}\|Q(s) x\| \leq N\left\|\Phi_{Q}(t, s) x\right\|$,

for all $(t, s, x) \in \Delta \times X$. 
Proof. Necessity. It results for $\nu=\min \{-\alpha, \beta\}$.

Sufficiency. It is immediate.

Definition 2.2. The constant $\nu$ from Proposition 2.1 is called dichotomy rate.

In the following proposition we consider the real constants

$$
\alpha<\beta, \gamma=\frac{\alpha+\beta}{2} \text { and } \delta=\frac{\beta-\alpha}{2}
$$

and the evolution operator $\Phi^{\gamma}: \Delta \rightarrow \mathcal{B}(X)$, defined by

$$
\Phi^{\gamma}(t, s)=e^{-\gamma(t-s)} \Phi(t, s) .
$$

Proposition 2.2. The pair $(\Phi, P)$ admits an uniform exponential splitting with the splitting rates $\alpha<\beta$ if and only if $\left(\Phi_{\gamma}, P\right)$ has an uniform exponential dichotomy with the dichotomy rate $\delta$.

Proof. Necessity. For all $(t, s, x) \in \Delta \times X$ the following relations hold:

$\left(u_{e} d_{1}\right)\left\|\Phi_{P}^{\gamma}(t, s) x\right\|=e^{-\gamma(t-s)}\left\|\Phi_{P}(t, s) x\right\| \leq N e^{-\delta(t-s)}\|P(s) x\| ;$

$\left(u e d_{2}\right) e^{\delta(t-s)}\|Q(s) x\| \leq N e^{(\delta-\beta)(t-s)}\left\|\Phi_{Q}(t, s) x\right\|=N\left\|\Phi_{Q}^{\gamma}(t, s) x\right\|$.

Sufficiency. We show that the inequalities from Definition 2.1 are verified:

$\left(u e s_{1}\right)\left\|\Phi_{P}(t, s) x\right\|=e^{\gamma(t-s)}\left\|\Phi_{P}^{\gamma}(t, s) x\right\| \leq N e^{\alpha(t-s)}\|P(s) x\| ;$

$\left(u e s_{2}\right) e^{\beta(t-s)}\|Q(s) x\|=e^{\gamma(t-s)} e^{\delta(t-s)}\|Q(s) x\| \leq N\left\|\Phi_{Q}(t, s) x\right\|$,

for all $(t, s, x) \in \Delta \times X$.

\section{Characterizations of Datko type for uniform expo- nential splitting}

In this section we consider an evolution operator $\Phi: \Delta \rightarrow \mathcal{B}(X)$ which is strongly measurable ( i.e. the mapping $t \mapsto\|\Phi(t, s) x\|$ is measurable for all $(t, s, x) \in \Delta \times X)$ and a family of projections $P: \mathbb{R}_{+} \rightarrow \mathcal{B}(X)$ which is invariant for $\Phi$.

A characterization for the uniform exponential splitting property is given by 
Theorem 3.1. The pair $(\Phi, P)$ admits an uniform exponential splitting if and only if there are the real constants $\omega>0, \gamma<\delta$ and $D \geq 1$ with:

$$
\begin{array}{ll}
\left(D e s_{1}\right) & \left\|\Phi_{P}(t, s) x\right\| \leq D e^{\omega(t-s)}\|P(s) x\| ; \\
\left(D e s_{2}\right) & e^{-\omega(t-s)}\|Q(s) x\| \leq D\left\|\Phi_{Q}(t, s) x\right\| ; \\
\left(D e s_{3}\right) & \int_{s}^{+\infty} e^{\gamma(s-\tau)}\left\|\Phi_{P}\left(\tau, t_{0}\right) x\right\| d \tau \leq D\left\|\Phi_{P}\left(s, t_{0}\right) x\right\| ; \\
& \int_{t_{0}}^{t} e^{\delta(t-\tau)}\left\|\Phi_{Q}\left(\tau, t_{0}\right) x\right\| d \tau \leq D\left\|\Phi_{Q}\left(t, t_{0}\right) x\right\|,
\end{array}
$$

for all $(t, s),\left(s, t_{0}\right) \in \Delta, x \in X$.

Proof. Necessity. It is a simple verification for

$$
\begin{gathered}
\omega=\left\{\begin{aligned}
\beta, & \text { if } \beta>0 \\
1, & \text { if } \beta=0 \\
-\beta, & \text { if } \beta<0,
\end{aligned}\right. \\
\gamma=\frac{\alpha+\beta}{2}, \delta=\frac{\alpha+3 \beta}{4} \text { and } D=\frac{4 N}{\beta-\alpha} .
\end{gathered}
$$

Sufficiency. We prove that the relations from Remark 2.2 are satisfied. Let $N=D^{2} e^{\omega} \max \left\{e^{-\gamma}, e^{\delta}\right\}$.

$\left(u e s_{1}^{\prime}\right)$ Case 1 . Let $(t, s),\left(s, t_{0}\right) \in \Delta$ with $t \geq s+1$. Then,

$$
\begin{gathered}
e^{-\gamma(t-s)}\left\|\Phi_{P}\left(t, t_{0}\right) x\right\|=\int_{t-1}^{t} e^{-\gamma(t-s)}\left\|\Phi_{P}\left(t, t_{0}\right) x\right\| d \tau \leq \\
\leq D \int_{t-1}^{t} e^{-\gamma(t-s)} e^{\omega(t-\tau)}\left\|\Phi_{P}\left(\tau, t_{0}\right) x\right\| d \tau \leq \\
\leq D e^{\omega} \max \left\{1, e^{-\gamma}\right\} \int_{s}^{+\infty} e^{\gamma(s-\tau)}\left\|\Phi_{P}\left(\tau, t_{0}\right) x\right\| d \tau \leq N\left\|\Phi_{P}\left(s, t_{0}\right) x\right\|,
\end{gathered}
$$

for all $s \geq t_{0}, t \geq s+1, x \in X$.

Case 2. Let $\left(s, t_{0}\right) \in \Delta, t \in[s, s+1]$. We have

$$
\left\|\Phi_{P}\left(t, t_{0}\right) x\right\| \leq D e^{\omega(t-s)}\left\|\Phi_{P}\left(s, t_{0}\right) x\right\| \leq
$$




$$
\begin{gathered}
\leq D e^{\omega} \max \left\{1, e^{-\gamma}\right\} e^{\gamma(t-s)}\left\|\Phi_{P}\left(s, t_{0}\right) x\right\| \leq \\
\leq N e^{\gamma(t-s)}\left\|\Phi_{P}\left(s, t_{0}\right) x\right\|,
\end{gathered}
$$

for all $s \geq 0, t \in[s, s+1], x \in X$.

We obtain that $\left(u e s_{1}^{\prime}\right)$ is verified for all $(t, s),\left(s, t_{0}\right) \in \Delta, x \in X$. $\left(\right.$ ues $\left._{2}^{\prime}\right)$ Case 1: We consider $\left(s, t_{0}\right) \in \Delta, t \geq s+1$. Then,

$$
\begin{gathered}
e^{\delta(t-s)}\left\|\Phi_{Q}\left(s, t_{0}\right) x\right\|=\int_{s}^{s+1} e^{\delta(t-s)}\left\|\Phi_{Q}\left(s, t_{0}\right) x\right\| d \tau \leq \\
\leq D \int_{s}^{s+1} e^{\omega(\tau-s)} e^{\delta(t-s)}\left\|\Phi_{Q}(\tau, s) \Phi_{Q}\left(s, t_{0}\right) x\right\| d \tau \leq \\
\leq D e^{\omega} \max \left\{1, e^{\delta}\right\} \int_{t_{0}}^{t} e^{\delta(t-\tau)}\left\|\Phi_{Q}\left(\tau, t_{0}\right) x\right\| d \tau \leq \\
\leq N\left\|\Phi_{Q}\left(t, t_{0}\right) x\right\| .
\end{gathered}
$$

It results

$$
e^{\delta(t-s)}\left\|\Phi_{Q}\left(s, t_{0}\right) x\right\| \leq N\left\|\Phi_{Q}\left(t, t_{0}\right) x\right\|,
$$

for all $\left(s, t_{0}\right) \in \Delta, t \geq s+1, x \in X$.

Case 2: Let $\left(s, t_{0}\right) \in \Delta, t \in[s, s+1]$. We obtain that

$$
\begin{aligned}
& e^{\delta(t-s)}\left\|\Phi_{Q}\left(s, t_{0}\right) x\right\| \leq D e^{\omega} \max \left\{1, e^{\delta}\right\}\left\|\Phi_{Q}\left(t, t_{0}\right) x\right\| \leq \\
& \leq N\left\|\Phi_{Q}\left(t, t_{0}\right) x\right\|,
\end{aligned}
$$

for all $\left(s, t_{0}\right) \in \Delta, t \in[s, s+1], x \in X$.

Thus, the relation $\left(u e s_{2}^{\prime}\right)$ is verified for all $(t, s),\left(s, t_{0}\right) \in \Delta, x \in X$.

In conclusion, the pair $(\Phi, P)$ admits an uniform exponential splitting.

As a particular case, we obtain a characterization of uniform exponential dichotomy given by

Corollary 3.1. The pair $(\Phi, P)$ has an uniform exponential dichotomy if 
and only if there are the constants $\omega, \mu>0$ and $D \geq 1$ with:

$$
\begin{array}{ll}
\left(\operatorname{Ded}_{1}\right) & \left\|\Phi_{P}(t, s) x\right\| \leq D e^{\omega(t-s)}\|P(s) x\| ; \\
\left(\operatorname{Ded}_{2}\right) & e^{-\omega(t-s)}\|Q(s) x\| \leq D\left\|\Phi_{Q}(t, s) x\right\| ; \\
\left(\operatorname{Ded}_{3}\right) & \int_{s}^{+\infty} e^{\mu(\tau-s)}\left\|\Phi_{P}\left(\tau, t_{0}\right) x\right\| d \tau \leq D\left\|\Phi_{P}\left(s, t_{0}\right) x\right\| ; \\
\left(\operatorname{Ded}_{4}\right) & \int_{t_{0}}^{t} e^{\mu(t-\tau)}\left\|\Phi_{Q}\left(\tau, t_{0}\right) x\right\| d \tau \leq D\left\|\Phi_{Q}\left(t, t_{0}\right) x\right\|,
\end{array}
$$

for all $(t, s),\left(s, t_{0}\right) \in \Delta, x \in X$.

Proof. Necessity. It is immediate, from Proposition 2.1, considering $\omega>$ $0, \mu \in(0, \nu)$.

Sufficiency. It results, using a similar technique with Theorem 3.1, for $\mu=\min \{-\gamma, \delta\}$.

\section{Lyapunov functions for uniform exponential split- ting}

Let $P: \mathbb{R}_{+} \rightarrow \mathcal{B}(X)$ be a family of projections on $X$ which is invariant for a strongly measurable evolution operator $\Phi: \Delta \rightarrow \mathcal{B}(X)$.

Definition 4.1. A mapping $L: \Delta \times X \rightarrow \mathbb{R}_{+}$is called Lyapunov function for the pair $(\Phi, P)$ if there exist the real constants $\alpha<\beta$ such that the following relations hold:

$$
\begin{aligned}
\left(L_{1}\right) \quad & L\left(t, s, \Phi_{P}\left(s, t_{0}\right) x\right)+\int_{s}^{t} e^{\alpha(s-\tau)}\left\|\Phi_{P}\left(\tau, t_{0}\right) x\right\| d \tau \leq \\
& \leq L\left(s, s, \Phi_{P}\left(s, t_{0}\right) x\right) ; \\
\left(L_{2}\right) \quad & \int_{s}^{t} e^{\beta(t-\tau)}\left\|\Phi_{Q}\left(\tau, t_{0}\right) x\right\| d \tau \leq L\left(t, t_{0}, Q\left(t_{0}\right) x\right),
\end{aligned}
$$

for all $(t, s),\left(s, t_{0}\right) \in \Delta, x \in X$.

A characterization of uniform exponential splitting property in terms of Lyapunov functions is given by 
Theorem 4.1. The pair $(\Phi, P)$ has an uniform exponential splitting if and only if there exist $L: \Delta \times X \rightarrow \mathbb{R}_{+}$a Lyapunov function for $(\Phi, P)$ and the constants $M \geq 1, \omega>0$ such that:

(i) $\left\|\Phi_{P}(t, s) x\right\| \leq M e^{\omega(t-s)}\|P(s) x\|$;

(ii) $e^{-\omega(t-s)}\|Q(s) x\| \leq M\left\|\Phi_{Q}(t, s) x\right\|$;

(iii) $L\left(s, s, \Phi_{P}\left(s, t_{0}\right) x\right) \leq M\left\|\Phi_{P}\left(s, t_{0}\right) x\right\|$;

(iv) $L\left(t, t_{0}, Q\left(t_{0}\right) x\right) \leq M\left\|\Phi_{Q}\left(t, t_{0}\right) x\right\|$,

for all $(t, s),\left(s, t_{0}\right) \in \Delta, x \in X$.

Proof. Necessity. Let $\alpha, \beta \in \mathbb{R}, \alpha<\beta$ and $L: \Delta \times X \rightarrow \mathbb{R}_{+}$,

$$
L(t, s, x)=\int_{t}^{+\infty} e^{\alpha(s-\tau)}\left\|\Phi_{P}(\tau, s) x\right\| d \tau+\int_{s}^{t} e^{\beta(t-\tau)}\left\|\Phi_{Q}(\tau, s) x\right\| d \tau .
$$

It is easy to verify that $L$ is a Lyapunov function for $(\Phi, P)$ and the conditions (iii) and (iv) are satisfied.

Sufficiency. Using Definition 4.1, $\left(L_{1}\right)$ and (iii) from the hypothesis we obtain

$$
\int_{s}^{t} e^{\alpha(s-\tau)}\left\|\Phi_{P}\left(\tau, t_{0}\right) x\right\| d \tau \leq L\left(s, s, \Phi_{P}\left(s, t_{0}\right) x\right) \leq M\left\|\Phi_{P}\left(s, t_{0}\right) x\right\|,
$$

and for $t \rightarrow+\infty$ it results that

$$
\int_{s}^{+\infty} e^{\alpha(s-\tau)}\left\|\Phi_{P}\left(\tau, t_{0}\right) x\right\| d \tau \leq M\left\|\Phi_{P}\left(s, t_{0}\right) x\right\|,
$$

for all $\left(s, t_{0}, x\right) \in \Delta \times X$.

Similarly, from $\left(L_{2}\right)$ and $(i v)$ we deduce

$$
\int_{t_{0}}^{t} e^{\beta(t-\tau)}\left\|\Phi_{Q}\left(\tau, t_{0}\right) x\right\| d \tau \leq M\left\|\Phi_{Q}\left(t, t_{0}\right) x\right\|
$$

for all $\left(t, t_{0}\right) \in \Delta, x \in X$.

From the relations (4.1), (4.2), (i), (ii) and Theorem 3.1 we obtain that $(\Phi, P)$ admits an uniform exponential splitting. 
As a particular case, we obtain a characterization for the uniform exponential dichotomy, given by

Corollary 4.1. The pair $(\Phi, P)$ has an uniform exponential dichotomy if and only if there exist $L: \Delta \times X \rightarrow \mathbb{R}_{+}$a Lyapunov function for $(\Phi, P)$ and the constants $M \geq 1, \omega>0$ such that:

(i) $\left\|\Phi_{P}(t, s) x\right\| \leq M e^{\omega(t-s)}\|P(s) x\|$;

(ii) $e^{-\omega(t-s)}\|Q(s) x\| \leq M\left\|\Phi_{Q}(t, s) Q(s) x\right\|$;

(iii) $L\left(s, s, \Phi_{P}\left(s, t_{0}\right) x\right) \leq M\left\|\Phi_{P}\left(s, t_{0}\right) x\right\|$;

(iv) $L\left(t, t_{0}, Q\left(t_{0}\right) x\right) \leq M\left\|\Phi_{Q}\left(t, t_{0}\right) x\right\|$,

for all $(t, s),\left(s, t_{0}\right) \in \Delta, x \in X$.

\section{References}

[1] B. Aulbach and J. Kalkbrenner, Exponential forward splitting for noninvertible difference equations, Comput. Math. Appl., 42, (2001), 743-754

[2] M. G. Babuţia and M. Megan, Exponential dichotomy concepts for evolution operators on the half-line, Ann. Acad. Rom. Sci. Ser. Math. Appl., 7, (2015), 209226

[3] A. J. G. Bento, N. Lupa, M. Megan, and C. Silva, Integral conditions for nonuniform $\mu$ - dichotomy, arXiv6v1 [math.DS], (2014)

[4] L. Biriş and M. Megan, On a concept of exponential dichotomy for cocycles of linear operators in Banach spaces, accepted for publication in B. Math. Soc. Sci. Math.

[5] R. Datko, Uniform Asymptotic Stability of Evolutionary Processes in a Banach Space, SIAM J. Math. Anal., 3, (1972), 428-445

[6] M. I. Kovacs, M. Megan, and C. L. Mihit, On $(h, k)$-dichotomy and $(h, k)$ trichotomy of noninvertible evolution operators in Banach spaces, Analele Universităţii de Vest din Timişoara, Seria Matematică-Informatică, LII, (2014), 127-143

[7] N. Lupa and M. Megan, Exponential dichotomies of evolution operators in Banach spaces, Monatsh. Math., 174, (2014), 265-284

[8] M. Megan, On $(h, k)$-dichotomy of evolution operators in Banach spaces, Dynam. Systems Appl., 5, (1996), 189-196

[9] M. Megan and M. G. Babuţia, Nonuniform exponential dichotomy for noninvertible evolution operators in Banach spaces, accepted for publication in An. Stiint. U. Al. I-Mat

[10] M. Megan, B. Sasu, and A. L. Sasu, On nonuniform exponential dichotomy of evolution operators in Banach spaces, Integr. Equat. Oper. Th., 44, (2002), 71-78 
[11] M. Megan and C. Stoica, Concepts of dichotomy for skew-evolution semiflows in Banach spaces, Ann. Acad. Rom. Sci. Ser. Math. Appl., 2, (2010), 125-140

[12] N. Van Minh, F. Räbiger, and R. Schnaubelt, Exponential stabi lity, exponential expansiveness and exponential dichotomy of evolution equations on the half-line, Integ. Equ. Oper. Th., 32, (1998), 332-353

[13] O. Perron, Die Stabilitätsfrage bei Differentialgleichungen, Math. Z., 32, (1930), 703-728

[14] I. L. Popa, M. Megan, and T. Ceauşu, Nonuniform exponential dichotomies in terms of Lyapunov functions for noninvertible linear discrete-time systems, The Scientific World Journal, (2013), Article ID 901026

[15] L. Zhou, K. Lu, and W. Zhang, Roughness of temperated exponential dichotomies for infinite-dimensional random difference equations, J. Differential Equations, 254, (2013), 4024-4046

Claudia Luminiţa Mihiţ

Department of Mathematics

West University of Timişoara

V. Pârvan Blvd., No. 4

300223 Timişoara

Romania

E-mail: mihit.claudia@yahoo.com

Codruţa Simona Stoica

Faculty of Exact Sciences

"Aurel Vlaicu" University

Elena Drăgoi Str., No. 2

310330 Arad

Romania

E-mail: codruta.stoica@uav.ro

Mihail Megan

Academy of Romanian Scientists

West University of Timişoara

V. Pârvan Blvd., No. 4

300223 Timişoara

Romania

E-mail: megan@math.uvt.ro

Received: 1.11.2015

Accepted: 15.12.2015 